\title{
Kinking of transfemoral central venous catheter in an infant: a case report
}

Volume 5 Issue 5 - 2016

\author{
Sapna Bansal \\ Maharishi Markandeshwar University, India
}

\section{Introduction}

Central venous cannulation (CVC) in infants and neonates is much more challenging especially in critically ill patients and in infants who fail to thrive. This procedure requires a lot of experience with modern equipments. Of all the sites for $\mathrm{CVC}$, femoral vein (FV) is an important site with fewer side effects but not free from it. Here we present a case of malpositioned central femoral catheter in a 2 month old infant weighing $2.5 \mathrm{kgs}$ posted for Kasai procedure.

\section{Case report}

A 2 month old infant weighing $2.5 \mathrm{kgs}$ was posted for Kasai procedure for extrahepatic biliary atresia. Because of major nature of the procedure and difficult peripheral cannulation CVC through femoral route was planned. A $4.5 \mathrm{~F}, 6 \mathrm{~cm}$ triple lumen central venous catheter was inserted through the right femoral vein using the Seldinger's technique in the first attempt without difficulty. A good back flow was confirmed and the catheter was secured and taped. During the surgery there was difficulty in aspiration of blood although fluid and blood infusions were smooth. Postoperatively chest X-ray was done which revealed kinking of the catheter in the right femoral vein. The fluid infusion was terminated and catheter was removed (Figure 1).



Figure I Showing kinking of central femoral catheter in right femoral vein.

\section{Discussion}

The main purpose of CVC in infants are major surgical procedure, difficult peripheral cannulation, administration of drugs, fluids, total parentral nutrition, blood and central venous pressure monitoring. The common sites of catheter insertion in infants are subclavian vein, internal jugular vein, femoral vein, umbilical vein or external jugular vein. The subclavian venous cannulation has complication rate of approximately $4 \%{ }^{1}$ which includes malpositioned catheter into the ipsilateral IJV or contralateral SCV, pneumothorax, haemothorax

\author{
Correspondence: Sapna Bansal, Maharishi Markandeshwar \\ University, Mullana, 289, durga colony, Naraingarh, India, Tel \\ 9896633 I25, Email drsapna 10@gmail.com \\ Received: July 16, 2016 | Published: September 14, 2016
}

and air embolism. Cardiac temponade leading to death has also been reported as a major complication with SCV, IJV or umbilical vein cannulation. Weil et al. ${ }^{2}$ found that the catheter tip in right atrium is associated with increased risk of cardiac tamponade. Kayashima $\mathrm{K}$ et al. ${ }^{3}$ evaluated the mechanical complications during paediatric CVC from 1994 to 2013 and found that most of these complications are associated with SCV, IJV, umbilical vein cannulation. In an another study done by Jha M et al. ${ }^{4}$ showed more complication rate with SCV cannulation as compared to FV cannulation in terms of more arterial puncture, hematoma and pneumothorax. Advantages of femoral cannulation includes large diameter of the vein, convenient access and distant to the cardiac and pulmonary structures. Accident arterial puncture can be easily managed by applying pressure. So in our case, we selected femoral route for central venous cannulation, but it also has inherent complications.

Malposition of femoral venous catheter into ascending lumbar vein was detected by Carrion et al. ${ }^{5}$ which was then repositioned using ultrasonographic guidance. Paravertebral and intraspinal malposition resulting in quardiplegia has been also reported in neonates. ${ }^{6}$ Therefore catheters in the ascending vein or vertebral plexus should be removed immediately. First warning signs of catheter malposition is absent blood on aspiration. Others are catheter present over vertebral column on x-ray and resistance to guide wire insertion. In our case we could not aspirate blood and were also unable to reinsert the guidewire. These malpositioned catheters can be identified by ultrasound, X-ray, linogram (contrast enhanced study) and fluroscopic screening. In our case X-ray abdomen revealed kinking of the catheter in the femoral vein. It could be due to defect in the guide wire material, structure or extra force applied on the soft guidewire of $4.5 \mathrm{~F}$ catheter size. The force applied should not be more than the inner strength of the guide wire. Onal et al. ${ }^{7}$ also reported a case of separation of spiral wire surrounding guide wire due to weakness and the inadequacy of the material used. Other complications of femoral cannulation are femoral artery puncture leading to hematoma, pseudoaneurysm, higher infection rate and peripheral embolism. ${ }^{4}$ Avascular necrosis can occur due to recurrent femur head injury. Most of these complications occurs with $\mathrm{CVC}$ using landmark technique. Therefore use of ultrasound for central venous cannulation can be a technique to reduce the complications. It clearly demonstrates the position with diameter of the vein and nearby surrounding structures. It also helps in detecting the puncture site and needle position in paediatric patients 
through the femoral route. Most of the studies have been done on IJV cannulation using ultrasound in paediatric patients. ${ }^{8}$ A meta-analysis was done on real time ultrasound guided (RTUS) CVC suggested that SCV and IJV cannulation can be benefited more as compared to FV cannulation in terms of reduced arterial punctures and hematoma where as it improves cannulation failures in patient receiving FV cannulation. ${ }^{9}$

US guided cannulation of the femoral vein in paediatric patients is superior to the landmark technique in terms of fewer attempts, complications and lesser time ${ }^{10}$ but studies regarding its role in detecting malpositioning and kinking are lacking. Aouad MT et al. ${ }^{11}$ also proved ultrasound guided FV cannulation superior to landmark technique. American Society of Echocardiography and the Society of Cardiovascular Anaesthesiologist recommend that trained clinicians use real time ultrasound during IJV and FV cannulation whenever possible to improve cannulation success and reduce the incidence of complications associated with insertion of large bore catheter in paediatric patients. ${ }^{12}$ The location of the catheter tip should be assessed immediately after insertion particularly in infants because these mechanical complications can be life threatening and in addition it requires prompt and appropriate management to ensure patient safety. Fluroscopic guided guidewire and catheter advancement during CVC can also reduce complications related to its misplacement.

\section{Conclusion}

In conclusion awareness regarding possible complications of central venous cannulation can help to reduce the unfavourable outcomes and improve patient's safety.

\section{Acknowledgments}

None.

\section{Conflicts of interest}

The authors declare there is no conflict of interests.

\section{Funding}

None.

\section{References}

1. Jonge RC, Polderman KH, Gemke RJ. Central venous catheter use in the pediatric patient:mechanical and infectious complications. Pediatr Crit Care Med. 2005;6(3):329-39.
2. Weil BR, Ladd AP, Yoder K. Pericardial effusion and cardiac tamponade associated with central venous catheters in children:an uncommon but serious and treatable condition. J Pediatr Surg. 2010;45(8):1687-1692.

3. Kayashima K, Imai K. Evaluation of mechanical complications during pediatric central venous catheter placement from 1994 to 2013. International Journal of Anesthesiology Research. 2013;1:36-46.

4. Jha M, Kumar VS, Bokil S, et al. Complications of central venous catheter cannulation in tertiary care hospital ICU, a 2 years retrospective, observational study. Pediatric Anesthesia and Critical Care Journal. 2013;1(2):87-92.

5. Carrion E, Hertzog JH, Gunter AW, et al. Misplacement of a femoral venous catheter into the ascending lumbar vein:repositioning using ultrasonographic guidance. Intensive Care Med. 2001;27(1):240-242.

6. Zenker M, Rupprecht T, Hofbeck M, et al. Paravertebral and intraspinal malposition of transfemoral central venous catheters in newborns. The Journal of Pediatrics. 2000;136(6):837-840.

7. Onal O, Çelebioğlu B, Kanbak M, et al. An unusual complication during femoral venous catheterization:Separation of spiral wire surrounding guide wire and its late diagnosis in a child. OA Case Reports. 2013;2:134.

8. Verghese ST, McGill WA, Patel RI, et al. Ultrasound-guided internal jugular venous cannulation in infants: a prospective comparison with the traditional palpation method. Anesthesiology. 1999;91(1):71-77.

9. Wu Sy, Ling Q, Cao LH, et al. Real time two-dimension guidance for central venous cannulation:a meta-analysis. Anesthesiology. $2013 ; 118(2): 361-375$.

10. Eldabaa AA, Elgebaly AS, Elhafz AAA, et al. Comparison of ultrasound-guided vs. anatomical landmark-guided cannulation of the femoral vein at the optimum position in infant. South Afr J Anaesth Analg. 2012;18:162-166.

11. Aouad MT, Kanazi GE, Abdallah FW, et al. Femoral Vein Cannulation performed by residents: a comparison between Ultrasound-guided and landmark technique in infants and children undergoing cardiac surgery. Anesth Analg. 2010;111(3):724-728.

12. Troianos CA, Hartman GS, Glas KE, et al. Guidelines for Performing Ultrasound Guided Vascular Cannulation:Recommendations of the American Society of Echocardiography and the Society of Cardiovascular Anesthesiologists. Anesth Analg. 2012;114(1):46-72. 